\title{
Glutathione levels influence chronological life span of Saccharomyces cerevisiae in a glucose-dependent manner
}

Mayra Fabiola Tello-Padilla ${ }^{1}$, Alejandra Yudid Perez-Gonzalez ${ }^{1}$, Melina Canizal-García ${ }^{1}$, Juan Carlos González-Hernández², Christian Cortes-Rojo ${ }^{3}$, Ivanna Karina Olivares-Marin ${ }^{4}$ and Luis Alberto Madrigal-Perez ${ }^{1 *}$

${ }^{1}$ Laboratorio de Biotecnología Microbiana, Instituto Tecnológico Superior de Ciudad Hidalgo, Av. Ing. Carlos Rojas Gutiérrez \#2120, Ciudad Hidalgo, Michoacán, 61100, México.

${ }^{2}$ Laboratorio de Bioquímica del Instituto Tecnológico de Morelia, Av. Tecnológico de Morelia, Morelia, Michoacán, 58030, México.

${ }^{3}$ Instituto de Investigaciones Químico Biológicas, Universidad Michoacana de San Nicolás de Hidalgo, Morelia, Michoacán, 58120, México.

${ }^{4}$ Universidad Autónoma de Querétaro, Cerro de las Campanas, Santiago de Querétaro, Qro, 76010, México.

*Address for correspondence:

Phone: +52 (786) 154-9000

Fax: +52 (786) 154-9000

E-mail: 1madrigal@itsch.edu.mx

Short Title: Life span of budding yeast depends on glutathione and glucose levels 


\begin{abstract}
Diet plays a key role in determining the longevity of the organisms since it has been demonstrated that glucose restriction increases lifespan whereas a high-glucose diet decreases it. However, the molecular basis of how diet leads to the aging process is currently unknown. We propose that the quantity of glucose that fuels respiration influences ROS generation and glutathione levels, and both chemical species impact in the aging process. Herein, we provide evidence that mutation of the gene GSH1 diminishes glutathione levels. Moreover, glutathione levels were higher with $0.5 \%$ than in $10 \%$ glucose in the gsh $1 \Delta$ and WT strains. Interestingly, the chronological life span (CLS) was lowered in the gsh1 $\Delta$ strain cultured with $10 \%$ glucose but not under dietary restriction. The $g \operatorname{sh} 1 \Delta$ strain also showed an inhibition of the mitochondrial respiration in 0.5 and $10 \%$ of glucose but only increased the $\mathrm{H}_{2} \mathrm{O}_{2}$ levels under dietary restriction. These results correlate well with the GSH/GSSG ratio, which showed a decrease in gsh $1 \Delta$ strain cultured with $0.5 \%$ glucose. Altogether these data indicate that glutathione has a major role in the function of electron transport chain (ETC) and is essential to maintain life span of Saccharomyces cerevisiae in $10 \%$ glucose.
\end{abstract}

\title{
Keywords
}

Aging; GSH; yeast; glucose concentration; oxidative stress; dietary restriction 


\section{Introduction}

Aging is an irreversible process that all the living organisms undergo. It has been postulated that aging leads to the malfunction of multiple cellular processes, which drives to chronic degenerative diseases (Barzilai et al., 2012). Diet is a chief environmental factor that influences the development of chronic degenerative diseases related to aging (Brandhorst et al., 2015; Honma et al., 2012; Prasad et al., 2012; Wei et al., 2017). Accordingly, dietary restriction increases the longevity across a wide range of species and has also been associated with the amelioration of some chronic degenerative diseases (Colman et al., 2009; Colman et al., 2014; Marchal et al., 2012), although it is not entirely clear how the restriction of certain nutrients such as glucose contributes to longevity extension. Saccharomyces cerevisiae is a useful model to elucidate the relationships between nutrient load and aging, as the effects of a high-energy diet and dietary restriction can be mimicked in this yeast by evaluating the effects of glucose concentrations of $>1 \% \mathrm{w} / \mathrm{v}$ or $<0.5 \% \mathrm{w} / \mathrm{v}$, respectively, on chronological lifespan (CLS), which is the viability of the cell culture in a certain time in a nondividing, quiescent-like condition (Kaeberlein, 2010; Madrigal-Perez et al., 2016; Rockenfeller and Madeo, 2010). High concentrations of glucose decrease CLS by a mechanism related to increased oxidative stress (Barros et al., 2004; Mesquita et al., 2010; Weinberger et al., 2010).

Glutathione ( $\gamma$-glutamyl-L-cysteinyl-glycine) is the most abundant thiol tripeptide and is involved in numerous cellular processes such as the control of redox environment, iron metabolism, protein stabilization, mitochondrial function, among others (Brasil et al., 2013; Kumar et al., 2011; Penninckx and Elskens, 1993). The levels of glutathione increase under dietary restriction (Rebrin et al., 2003; Sharma et al., 2010; Walsh et al., 2014). Accordingly, deletion of SCH9, TOR1, and RAS2 nutrient-sensing genes exhibits a dietary restriction phenotype and maintain high levels of reduced glutathione when are exposed to stress, besides displaying enhanced survival to the anti-cancer agent cisplatin by using glutathione to detoxify this drug (Mariani et al., 2014). Overall, these findings suggest that glutathione levels are regulated by glucose or other nutrients and may have a fundamental role during cellular stress.

Glutathione is synthesized in a two-step process catalyzed by the proteins encoded by the genes GSH1 and GSH2 (Tang et al., 2015). The mutation in S. cerevisiae of the gene GSH1 (gsh1 $\Delta$ ) dramatically decreases glutathione levels from approximately $4 \mathrm{mM}$ to $0.03 \mathrm{mM}$ and increases yeast sensitivity to $\mathrm{H}_{2} \mathrm{O}_{2}$ (Hatem et al., 2014). The gshl $\Delta$ mutation also reverted the resistance of $S$. cerevisiae to the anti-cancer compound cisplatin under dietary restriction (Mariani et al., 2014).

Considering that dietary restriction increases glutathione levels and extends longevity and that glutathione optimizes the mitochondrial function, the aim of this work was to elucidate whether longevity extension by dietary restriction is mediated by an improvement of mitochondrial function via an enhancement of both the redox status and the concentration of glutathione. 


\section{Material and methods}

\section{Strains}

The BY4741 wild type strain of S. cerevisiae (MATa; his3 11 ; leu2 $\Delta 0$; met15 00 ; ura3 $\Delta 0$;

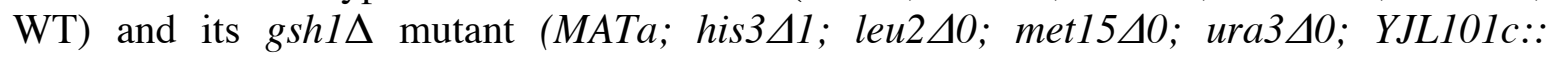
KanMX4; gsh1A) were acquired from EUROSCARF (Frankfurt, Germany). The strains were maintained in yeast extract-peptone-dextrose (YPD) medium (1\% yeast extract, $2 \%$ casein peptone and $2 \%$ glucose). Medium for the mutant strain was supplemented with 150 $\mu \mathrm{g} / \mathrm{mL}$ of G-418 disulfate salt (Sigma-Aldrich, St. Louis, MO, USA).

\section{Chronological life span assay}

The CLS was performed according to the protocol described by Murakami et al. (2008) as follow. WT and gshl $\Delta$ cells were cultured from frozen stocks by plating onto YPD agar, consisting of $1 \%$ yeast extract (Sigma-Aldrich), 2\% casein peptone (Sigma-Aldrich), $2 \%$ bacteriological agar (Affymetrix, Santa Clara, CA, USA) and supplemented with $2 \%$ of glucose. The plates were incubated at $30^{\circ} \mathrm{C}$ for $48 \mathrm{~h}$ or until single colonies appear. Then, three singles colonies were picked, and cultured overnight in $2 \mathrm{~mL}$ YPD medium supplemented with $2 \%$ glucose. To induce aging in the cultures, $300 \mu \mathrm{L}$ of the fresh overnight culture were placed into $3 \mathrm{~mL}$ YPD $(0.5,2$ or $10 \%$ glucose $)$ medium in a $15 \mathrm{~mL}$ test tube to keep 1:5 liquid-air ratios. Cultures were maintained at $30{ }^{\circ} \mathrm{C}$ with constant agitation at $180 \mathrm{rpm}$ throughout the experiment. After incubation for 2 days, $500 \mu \mathrm{L}$ aliquots were taken from aging cultures and inoculated into $5 \mathrm{~mL}$ YPD medium supplemented with $2 \%$ glucose in a $25 \mathrm{~mL}$ Erlenmeyer flask. Cultures were incubated at 30 ${ }^{\circ} \mathrm{C}$ for $12 \mathrm{~h}$ in a shaking incubator (MaxQ 4000, Thermo Scientific, MA, USA) at $180 \mathrm{rpm}$. The absorbance of the cultures at $600 \mathrm{~nm}$ was measured every hour during a period of $12 \mathrm{~h}$ using a UNICO spectrophotometer (UNICO, NJ, USA). The growth kinetics procedure was repeated every two days. To determine the survival percentage, the doubling time $(t d)$ was calculated by adjusting the growth curves with the exponential growth equation using the software GraphPad Prism 6.00 (GraphPad Software, La Jolla, CA, USA). Additionally, the time shift $(\Delta t n)$ was obtained by calculating the natural logarithm of the optic density at 600 $\mathrm{nm}\left(\mathrm{OD}_{600}\right)$ obtained at the exponential phase of the growth curve and then interpolating at $\mathrm{OD}_{600}=0.5$ using linear regression. Finally, the survival percentage $(S n)$ was calculated according to the following equation:

$S n=\frac{1}{2^{\left(\frac{\Delta t n}{t d}\right)}} \times 100$

On the other hand, the area under curve (AUC) survival was computed from the data of survival percentage vs. time using the trapezoid rule in the GraphPad Prism 6.00.

\section{Determination of in situ mitochondrial respiration}

The oxygen consumption was measured polarographically at $28{ }^{\circ} \mathrm{C}$ using a Clark-type oxygen electrode (YSI 5300, Yellow Springs, OH, USA). S. cerevisiae cultures were harvested either at mid-log phase (D.O. ${ }_{600} \sim 0.5$ ) or after six days of inoculation (chronologically aged yeast culture) at $5000 \times \mathrm{g}$ for 5 minutes at $28{ }^{\circ} \mathrm{C}$. Then, $125 \mathrm{mg}$ of cells were resuspended in $5 \mathrm{~mL}$ of buffer $10 \mathrm{mM} 2-(\mathrm{N}$-morpholino) ethane sulfonic acid 
(MES-TEA; Sigma-Aldrich), adjusted to pH 6.0 with triethanolamine (Sigma-Aldrich) in a closed chamber with constant stirring. The basal respiration was determined using $10 \mathrm{mM}$ glucose as substrate. The maximal rate of respiration was evaluated by adding $10 \mu \mathrm{M}$ of the uncoupler carbonyl cyanide 3-chlorophenylhydrazone (CCCP). Non-mitochondrial oxygen consumption was discriminated by adding $10 \mu \mathrm{M}$ Antimycin $\mathrm{A}$ at the end of each determination. Oxygen consumption was expressed as nanoatoms $\mathrm{O}_{2}$ per minute per milligram of cells.

\section{Quantification of $\mathrm{H}_{2} \mathrm{O}_{2}$ release}

Superoxide anion produced in the mitochondrial matrix is rapidly dismutated by the manganese-dependent superoxide dismutase ( $\mathrm{Sod} 2 \mathrm{p})$ to hydrogen peroxide $\left(\mathrm{H}_{2} \mathrm{O}_{2}\right)$, which diffuses through mitochondrial and plasmatic membranes to the surrounding medium (Dikalov and Harrison, 2014). Thereby, the extracellular $\mathrm{H}_{2} \mathrm{O}_{2}$ release was used as an indicator of ROS levels and was determined by the Amplex red hydrogen peroxide assay kit (Invitrogen, Waltham, MA, USA) according to the manufacturer's instructions. Briefly, overnight $S$. cerevisiae cultures were harvested at $5000 \times g$ for 5 minutes at $28^{\circ} \mathrm{C}$. The cells were washed three times with deionized water and resuspended in $2 \mathrm{~mL}$ of assay buffer containing $20 \mathrm{mM}$ Tris- $\mathrm{HCl}, 0.5 \mathrm{mM}$ EDTA and $2 \%$ ethanol at $\mathrm{pH}$ 7. The cells were placed in 96 well black plates at a density of $3 \times 10^{6}$ cells/well. Then, $50 \mu \mathrm{L}$ of work solution (100 $\mu \mathrm{M}$ Amplex red and $10 \mathrm{U} / \mathrm{mL}$ horseradish peroxidase) was added to each well and incubated for $30 \mathrm{~min}$ at $30^{\circ} \mathrm{C}$ with constant agitation in a horizontal shaker and protected from light. Finally, the basal release of $\mathrm{H}_{2} \mathrm{O}_{2}$ was measured at an excitation wavelength of $563 \mathrm{~nm}$ and an emission wavelength of $587 \mathrm{~nm}$ with a microplate reader (Varioskan Flash Multimode Reader, Thermo-Scientific).

\section{Quantification of glutathione}

Total glutathione, which comprises the sum of the concentrations of reduced glutathione (GSH) plus oxidized glutathione (GSSG), was quantified by measuring the reduction of 5,5 ' -dithio-bis(2-nitrobenzoic acid) (DTNB) (Rahman et al., 2006; Tietze, 1969). $3 \mathrm{~mL}$ of exponential phase cultures $\left(\mathrm{OD}_{600} \sim 0.5\right)$ or chronologically aged cultures (six days after inoculation) were harvested at $5000 \mathrm{x} g$ for $2 \mathrm{~min}$. Cells were normalized to an $\mathrm{OD}_{600} \sim 0.5$, washed twice with $2 \mathrm{~mL}$ PBS, resuspended in $25 \mu \mathrm{L}$ of PBS and homogenized by vortexing with glass beads. Then, the cytosolic extracts were deproteinized with 5-sulfosalicylic acid $5 \%$ (Sigma-Aldrich) at $-20^{\circ} \mathrm{C}$ for $30 \mathrm{~min}$. Glutathione determination reaction was prepared using $700 \mu \mathrm{L}$ buffer KPE (0.1 M phosphate potassium, 0.001 M EDTA), $100 \mu \mathrm{L}$ cell extract, $60 \mu \mathrm{L}$ glutathione reductase solution (6 U/mL; Sigma-Aldrich), $60 \mu \mathrm{L}$ of 0.66 $\mathrm{mg} / \mathrm{mL}$ DTNB solution and $60 \mu \mathrm{L}$ of $0.66 \mathrm{mg} / \mathrm{mL} \beta-\mathrm{NADPH}$ solution (Sigma-Aldrich). Finally, the samples were read at $412 \mathrm{~nm}$ using a microplate reader (Varioskan Flash Multimode Reader, Thermo-Scientific). For GSSG determination, the cell extracts were treated with 4-vinyl pyridine (Sigma-Aldrich) for GSH derivatization, and the excess of 4vinyl pyridine was neutralized with triethanolamine. Then, GSSG was quantified by reducing it with glutathione reductase as described above.

\section{Statistical analyses}

Two-tailed unpaired Student $t$-test was applied to test for differences in CLS, $\mathrm{H}_{2} \mathrm{O}_{2}$ release, total glutathione concentration and rate of oxygen consumption. For all analyses, at least 3 
independent experiments were performed. Statistical analyses were computed using GraphPad Prism 6.00 (GraphPad Software, La Jolla California, USA).

\section{Results}

Effect of glucose concentration on $\mathrm{H}_{2} \mathrm{O}_{2}$ levels and mitochondrial function in the exponential growth phase and its impact over CLS

As shown in the Fig. 1a, 10\% glucose decreases the yeast CLS severely, while dietary restriction, which was mimicked by adding $0.5 \%$ glucose to the culture media, increases in a notable way the CLS, as reflected by a full preservation of cell viability all over the experiment. Antimycin A-sensitive respiration and $\mathrm{H}_{2} \mathrm{O}_{2}$ release were measured to assess if the effects of excessive glucose were related to differences in the exponential growth phase (i.e. before the beginning of chronological aging) in mitochondrial function and ROS production, respectively. It can be observed a $60 \%$ increase of $\mathrm{H}_{2} \mathrm{O}_{2}$ levels in the yeast growing with $10 \%$ glucose in comparison to yeast with $0.5 \%$ glucose (Fig. 1b), as well a severe repression of respiration in both state 4 (i.e. basal respiration) and uncoupled (U) state (i.e. maximal respiration rate with CCCP). Besides, respiration in cells under dietary restriction was almost entirely uncoupled as the increase of oxygen consumption elicited by CCCP was negligible (Figs. 1c and d). Therefore, these results suggest that excessive concentrations of glucose during the exponential growth set early defects in mitochondrial function that may be related to exacerbated ROS production and/or antioxidant depletion that contribute to the phenotype of impaired CLS.

Effect of GSH1 mutation on glutathione levels at the exponential growth phase

The levels of glutathione in the WT cells and the gshl $\Delta$ mutant were compared at three concentrations of glucose in order to explore if the levels of this antioxidant at the exponential growth phase are influenced by the concentration of glucose. As expected, total glutathione content decreased $\sim 50 \%$ in the gsh $1 \Delta$ strain in comparison to WT cells at each level of glucose tested (Fig. 2). It is also important to mention that the $g \operatorname{sh} 1 \Delta$ strain is glutathione auxotroph and incapable of growing in minimal media. For this reason, we have used YPD medium. It is pertinent to note that in the mutant strain the glutathione was not completely depleted and this may be due to the YPD media glutathione content. 10\% glucose decreased glutathione concentration in the WT cells at similar levels to that observed in the gsh $1 \Delta$ mutant at $0.5 \%$ and $2 \%$ glucose. Regarding to the gsh $1 \Delta$ mutant, a $\sim 50 \%$ decrease in comparison to WT was observed only a $10 \%$ of glucose. These data suggest that compromised CLS may be the result of impaired mitochondrial function and excessive ROS production due to severe diminution of glutathione at the exponential growth phase.

\section{Influence of the GSH1 mutation on both $\mathrm{H}_{2} \mathrm{O}_{2}$ levels and CLS}

The effect of the GSH1 mutation on $\mathrm{H}_{2} \mathrm{O}_{2}$ levels was tested under dietary restriction and both standard and high-glucose levels. As seen in the Fig 3, ROS levels remained high in the gsh $1 \Delta$ mutant irrespectively of the glucose concentration tested. In contrast, a $\sim 40 \%$ decrease in ROS levels was observed in the WT strain under dietary restriction when compared to the gsh1 $\Delta$ mutant. These data suggest that intact glutathione synthesis is required for the decrement of ROS levels elicited by dietary restriction. 
The Fig 4 shows the CLS of both the WT and gsh1 $\Delta$ strains. At high-glucose level (i.e. $10 \%$ ) a drastic decrease of CLS was detected in the gsh1 $\Delta$ mutant in comparison to the WT cells. However, no differences in CLS were observed among the WT cells and the gsh1 mutant at the standard-glucose level and under dietary restriction. On the other hand, CLS was tested in the presence of $9.5 \%$ sorbitol, a non-metabolizable carbohydrate plus $0.5 \%$ glucose to discard that decreased CLS in the gsh1 $\Delta$ mutant was due to an osmotic effect instead of a result of nutrient overload. As expected, this approach caused a longer CLS in the gsh $1 \Delta$ mutant the exposure to $10 \%$ glucose (Fig. 5). This result indicates that decreased CLS in the gsh $1 \Delta$ mutant is not attributable to an osmotic stress elicited by the inability of this strain to synthesize glutathione.

Supplementation of the culture media with $100 \mu \mathrm{M}$ glutathione was carried out to assess what extent the diminution of the CLS observed in the gsh $1 \Delta$ mutant at $10 \%$ glucose was the consequence of decreased glutathione content. Glutathione addition increased the CLS of the gsh $1 \Delta$ mutant at the same level than the WT strain at $10 \%$ glucose (Fig. 5).

\section{Influence of GSH1 mutation over the mitochondrial function of yeast during CLS}

The in situ mitochondrial respiration of $S$. cerevisiae was measured to determine if the decrease of the CLS of gshl 1 mutant at $10 \%$ glucose was the result of impaired mitochondrial function.

In the exponential phase cultures, a severe decrease in both state 4 and $U$ states was found in the $g \operatorname{sh} 1 \Delta$ strain at 0.5 and $10 \%$ glucose in comparison to the WT cells (Fig. 6). Indeed, oxygen release was observed at $10 \%$ glucose in the $g \operatorname{sh} 1 \Delta$ strain, which is indicative of exacerbated ROS production. In contrast, no differences were seen in the respiratory states of both WT and gsh $1 \Delta$ cells at $2 \%$ glucose (Fig. 6). Unexpectedly, it was observed that sorbitol augmented several folds the respiratory rates in both state 4 and state $U$ when compared to the gsh $1 \Delta$ mutant growth only with $0.5 \%$ glucose, although respiration in $\mathrm{U}$ state did not reach the levels observed in the WT cells growth also with $0.5 \%$ glucose and 9.5\% sorbitol (Fig. 6). It is also remarkable that this treatment elicited a more uncoupled respiration in the $g \operatorname{sh} 1 \Delta$ mutant than in the WT cells as the ratio state U/state 4 was $\sim 2$ and $\sim 3$, respectively.

Regarding to mitochondrial function during chronological aging, both WT and $g \operatorname{sh} 1 \Delta$ cells exhibited a full inhibition of respiration in any respiratory state with $10 \%$ glucose, being observed instead oxygen release to the medium (Fig. 6). Also, no differences were detected in any respiratory state at $2 \%$ glucose; however, a $50 \%$ decrease in respiration was observed in the gsh $1 \Delta$ mutant with respect to WT cells under dietary restriction. On the other hand, divergent results were obtained when sorbitol was added during dietary restriction, since the respiration of the WT strain increased several times at levels even higher than that observed in the exponential phase, while in the gsh $1 \Delta$ mutant, sorbitol severely depressed respiration at the lower levels among all the experimental conditions (Fig. 6). Overall, these results suggest that either during the exponential phase or the chronological aging, GSH1 is needed to achieve an optimal mitochondrial function during dietary restriction, but it has not influence at higher concentrations of glucose. 


\section{Effects of GSH1 mutation on oxidative stress during CLS}

To explore whether enhanced oxidative stress due to GSH1 mutation is involved in the shortening of yeast CLS in high glucose we measured the GSH/GSSG ratio. As expected, oxidative stress augmented drastically in WT cells by the addition of $10 \%$ glucose in comparison to dietary restriction, as the GSH/GSSG ratio was more than twice lower at $10 \%$ glucose than at $0.5 \%$ (Fig. 7). In the gsh $1 \Delta$ mutant, GSH/GSSG ratio was severely diminished under dietary restriction in comparison to the WT strain, but non-statistically significant differences were observed among these strains at $10 \%$ glucose. These data suggest that intact glutathione synthesis is required to keep oxidative stress at minimum only during calorie restriction.

\section{Discussion}

Delaying of the aging process is critical to counteract the onset of age-related diseases and preserve health span (Barzilai et al., 2012). However, the molecular basis of the aging process is currently unknown. Restriction of certain nutrients such as glucose and supplementation with resveratrol improve the longevity of a wide range of species and have also been related with the amelioration of some chronic degenerative diseases (Huffman et al., 2016). Resveratrol and dietary restriction affect the mitochondrial function and the antioxidant systems, which directly impact ROS generation (Barros et al., 2004; MadrigalPerez et al., 2016; Plauth et al., 2016). Nonetheless, it is unclear whether the molecular mechanism by which dietary restriction counteract oxidative stress also modulates mitochondrial function and the aging process. Thereby, the aim of this study was to determine whether the amount of glucose in the culture medium influences ROS generation and glutathione levels, and its relationship with the aging process.

The mutant gsh1 $\Delta$ of $S$. cerevisiae exhibited diminished glutathione levels independently of the concentration of glucose (Fig. 2), while under dietary restriction (i.e. $0.5 \%$ glucose), displayed increased $\mathrm{H}_{2} \mathrm{O}_{2}$ levels (Fig. 3), impaired mitochondrial function either during the exponential growth or during chronological aging (Fig. 6) and enhanced oxidative stress with respect to WT cells (Fig. 7). Nevertheless, its chronological longevity was severely affected only in the presence of $10 \%$ glucose (Fig. 4). On the contrary, the effects of glucose on WT cells show a dependence on glucose concentration as seen with glutathione concentrations (Fig. 2), $\mathrm{H}_{2} \mathrm{O}_{2}$ levels (Fig. 3), mitochondrial function (Fig. 6) and oxidative stress (Fig. 7). It was discarded that the deleterious effects of $10 \%$ glucose over these parameters were due to an osmotic effect, by supplementing $9.5 \%$ sorbitol in the culture with 0.5 glucose (Figs. 5 and 6). However, the most surprising finding from these data is that sorbitol enhanced several times the respiration in the WT cells during chronological aging and, at lower extent, in the logarithmic phase, although in the gsh $1 \Delta$ mutant, sorbitol decreased respiration at any respiratory state (Fig. 6) without affecting CLS (Fig. 5). Osmotic stress activates a myriad of responses via the high-osmolarity glycerol (HOG) pathway (Brewster et al., 1993), among which, augmented catalase $\mathrm{T}$ activity is one of them (Schüller et al., 1994). Besides, it has been described that high osmolarity by $\mathrm{NaCl}$ increases glutathione synthesis (Jamnik et al., 2006). Assuming that osmotic stress by sorbitol also induces glutathione synthesis, it can be proposed that enhanced respiration was due to enhanced protection against the deleterious effects of aging and glucose on the ETC conferred by the synergic action of the antioxidant responses mounted by both dietary 
restriction and osmotic stress. In this regard, ROS like $\mathrm{H}_{2} \mathrm{O}_{2}$ decreases the activity of the complex I in mammalian mitochondria via glutathionylation of free cysteine residues as the concentration of oxidized glutathione increases (Taylor et al., 2003). Although S. cerevisiae does not contain a canonical complex I, it has been demonstrated that complexes II and III from yeast mitochondria are inhibited by $\mathrm{H}_{2} \mathrm{O}_{2}$ and that this effect can be reverted by a thiol reductant (Cortes-Rojo et al., 2007). Thus, the discrete effect of sorbitol on the respiration of the gsh $1 \Delta$ mutant may be related to that this strain cannot mount a response dependent on glutathione, which might drive to a higher inhibition of the ETC. However, the residual respiratory activity seen in this mutant with sorbitol instead the release of oxygen detected in the absence of this osmolyte may be related to a partial protective effect on other antioxidant systems like catalase as referred above. Concerning to the release of oxygen observed at $10 \%$ of glucose either at the exponential phase or during chronological aging, this can be interpreted as the result of enhanced superoxide radical formation, as the superoxide formed due to electron leak from respiration and its reaction with oxygen yields $\mathrm{H}_{2} \mathrm{O}_{2}$, which, in turn, is discomposed into $\mathrm{O}_{2}$ and $\mathrm{H}_{2} \mathrm{O}$ by catalase. On this basis, it can be proposed that although causing different longevity, $10 \%$ glucose elicited ROS production at similar levels in both WT and $g s h 1 \Delta$ cells because of a fully dysfunctional ETC.

GSH1 mutation affected ROS levels, mitochondrial function, and oxidative stress at $0.5 \%$ glucose without having any effect on these parameters at higher glucose concentrations, which suggest that glutathione is essential to preserve mitochondrial function, low ROS production and moderate oxidative stress under dietary restriction. However, it seems that enhanced mitochondrial dysfunction and ROS overproduction caused by low glutathione levels do not have an influence on longevity under dietary restriction since the GSHI mutation did not affect the CLS at $0.5 \%$ glucose but it did it at $10 \%$ glucose. Therefore, it can be hypothesized that other events influenced by glutathione may be responsible for the decrease in CLS at $10 \%$ glucose. One probable candidate is the production of excessive quantities of methylglyoxal, a toxic by-product of glycolysis that is highly toxic for cells because of their deleterious effects on DNA and proteins (Vander Jagt et al., 1992). Excessive methylglyoxal is detoxified in part by glyoxalase I, which converts methylglyoxal into S-D-lactoylglutathione in the presence of glutathione (Inoue and Kimura, 1996). Therefore, decreased glutathione synthesis may be disrupting this adaptive pathway and enhancing the deleterious effect of methylglyoxal, which should be expected to be occurring because of the high (i.e. 10\%) concentration of glucose in the medium. This is also in agreement with the protection conferred by the exogenous addition of $100 \mu \mathrm{M}$ glutathione against the deleterious effects of $10 \%$ glucose on the CLS of the gsh $1 \Delta$ mutant (Fig 5). Another probable process affecting CLS due to glutathione depletion is the synthesis of iron-sulfur clusters. In this concern, it has been demonstrated that glutathione is important for the maturation of iron sulfur clusters (Kumar et al., 2011). Indeed, the effect of GSH1 mutation upon iron sulfur proteins may be a cause of the impairment in the mitochondrial respiration observed in this study and the reason for the decrease in the respiratory metabolism in gsh $1 \Delta$ mutant cells grown under dietary restriction (Mannarino et al., 2008). Despite these arguments, it must be taken into account that fermentative metabolism because the culture of yeast in glucose as a carbon source might be buffering the deleterious effects of excessive glucose over mitochondrial function. Thus, the effect of 
the GSH1 mutation on CLS and its relationship with mitochondrial dysfunction and oxidative stress deserves further investigation using a non-fermentable carbon source.

Metabolic alterations characterizing metabolic syndrome have been related to glucoseinduced oxidative stress (Johnson et al., 2013). Augmented glycolytic flux increases the $\mathrm{NADH} / \mathrm{NAD}^{+}$ratio and enhances the reduction of the electron carries from the ETC. This augments mitochondrial membrane potential, which decreases the rate of electron transfer through the ETC leading to electron leak and exacerbated ROS generation. On the contrary, dietary restriction limits the amount of reduced electron carriers and decreases mitochondrial membrane potential, which increases the rate of electron transport to restore the mitochondrial membrane potential, being the final result a more rapid oxygen consumption and lower electron leak and attenuated ROS generation (Murphy, 2009). In agreement with this idea, the data from this study in the WT strain shows that there is an inverse relationship between the concentration of glucose and the rate of mitochondrial respiration, while higher $\mathrm{H}_{2} \mathrm{O}_{2}$ levels were detected, as the concentration of glucose was incremented.

The data from the gshl $\Delta$ mutant contradicts the role of mitochondrial dysfunction and excessive ROS generation in the decreased CLS of yeast at $10 \%$ glucose. However, the notable increase in $\mathrm{H}_{2} \mathrm{O}_{2}$, the almost full suppression of mitochondrial respiration and the depletion of glutathione at levels comparable to the gsh $1 \Delta$ mutant observed in the WT cells at $10 \%$ glucose during the exponential phase (Figs. 1 and 2), might set yeast for a more unfavorable redox environment decreasing its probabilities to survive during chronologic aging, which is in agreement with the notion that in post-diauxic cultures ROS generation occurs inversely to the CLS (Ramos-Gomez et al., 2017).

Despite dietary restriction decreases the levels of oxidative stress in the majority of organisms, it is known that this manipulation has a negligible effect on antioxidant systems, except on glutathione concentration, which remained high in the majority of studies about this issue (Walsh et al., 2014). The data of this report are in agreement with these studies since it was found that glutathione concentration and GSH/GSSG ratio were greater under dietary restriction than in high-glucose level.

Together, these data show that glutathione disturbs CLS in a glucose-dependent manner. Besides, these results provide further insight about the role of glucose in the CLS and its relationship with ROS generation and glutathione redox state.

\section{Acknowledgments}

The authors would like to thank Minerva Ramos-Gomez for their technical support. This work was supported by grants from Instituto Tecnológico Superior de Ciudad Hidalgo (3308.100310) and Tecnológico Nacional de México (165.14.2-PD and 166.14.2-PD).

\section{Conflict of Interest}

The authors declare no competing financial interest.

\section{References}


Barros M H, Bandy B, Tahara E B, et al. 2004. Higher respiratory activity decreases mitochondrial reactive oxygen release and increases life span in Saccharomyces cerevisiae. J Biol Chem 279, 49883-8.

Barzilai N, Huffman D M, Muzumdar R H, et al. 2012. The critical role of metabolic pathways in aging. Diabetes 61, 1315-22.

Brandhorst S, Choi I Y, Wei M, et al. 2015. A Periodic Diet that Mimics Fasting Promotes Multi-System Regeneration, Enhanced Cognitive Performance, and Healthspan. Cell Metab 22, 86-99.

Brasil A A, Belati A, Mannarino S C, et al. 2013. The involvement of GSH in the activation of human Sod1 linked to FALS in chronologically aged yeast cells. FEMS Yeast Res 13, 433-40.

Brewster J L, de Valoir T, Dwyer N D, et al. 1993. An osmosensing signal transduction pathway in yeast. Science 259, 1760-3.

Colman R J, Anderson R M, Johnson S C, et al. 2009. Caloric restriction delays disease onset and mortality in rhesus monkeys. Science 325, 201-4.

Colman R J, Beasley T M, Kemnitz J W, et al. 2014. Caloric restriction reduces age-related and all-cause mortality in rhesus monkeys. Nat Commun 5, 3557.

Cortes-Rojo C, Calderon-Cortes E, Clemente-Guerrero M, et al. 2007. Electron transport chain of Saccharomyces cerevisiae mitochondria is inhibited by $\mathrm{H} 2 \mathrm{O} 2$ at succinatecytochrome c oxidoreductase level without lipid peroxidation involvement. Free Radic Res 41, 1212-23.

Dikalov S I and Harrison D G. 2014. Methods for detection of mitochondrial and cellular reactive oxygen species. Antioxid Redox Signal 20, 372-82.

Hatem E, Berthonaud V, Dardalhon M, et al. 2014. Glutathione is essential to preserve nuclear function and cell survival under oxidative stress. Free Radic Biol Med 67, 103-14.

Honma T, Shinohara N, Ito J, et al. 2012. High-fat diet intake accelerates aging, increases expression of Hsd11b1, and promotes lipid accumulation in liver of SAMP10 mouse. Biogerontology 13, 93-103.

Huffman D M, Schafer M J and LeBrasseur N K. 2016. Energetic interventions for healthspan and resiliency with aging. Exp Gerontol 86, 73-83.

Inoue Y and Kimura A. 1996. Identification of the structural gene for glyoxalase I from Saccharomyces cerevisiae. J Biol Chem. 271, 25958-65.

Jamnik P, Medved P and Raspor P. 2006. Increased glutathione content in yeast Saccharomyces cerevisiae exposed to $\mathrm{NaCl}$. Ann Microbiol 56, 175-178.

Johnson S C, Rabinovitch P S and Kaeberlein M. 2013. mTOR is a key modulator of ageing and age-related disease. Nature 493, 338-45.

Kaeberlein M. 2010. Lessons on longevity from budding yeast. Nature 464, 513-9.

Kumar C, Igbaria A, D'Autreaux B, et al. 2011. Glutathione revisited: a vital function in iron metabolism and ancillary role in thiol-redox control. EMBO J 30, 2044-56.

Madrigal-Perez L A, Canizal-Garcia M, Gonzalez-Hernandez J C, et al. 2016. Energydependent effects of resveratrol in Saccharomyces cerevisiae. Yeast 33, 227-34.

Mannarino S C, Amorim M A, Pereira M D, et al. 2008. Glutathione is necessary to ensure benefits of calorie restriction during ageing in Saccharomyces cerevisiae. Mech Ageing Dev 129, 700-5. 
Marchal J, Blanc S, Epelbaum J, et al. 2012. Effects of chronic calorie restriction or dietary resveratrol supplementation on insulin sensitivity markers in a primate, Microcebus murinus. PLoS One 7, e34289.

Mariani D, Castro F A, Almeida L G, et al. 2014. Protection against cisplatin in calorierestricted Saccharomyces cerevisiae is mediated by the nutrient-sensor proteins Ras2, Tor1, or Sch9 through its target glutathione. FEMS Yeast Res 14, 1147-59.

Mesquita A, Weinberger M, Silva A, et al. 2010. Caloric restriction or catalase inactivation extends yeast chronological lifespan by inducing $\mathrm{H} 2 \mathrm{O} 2$ and superoxide dismutase activity. Proc Natl Acad Sci U S A 107, 15123-8.

Murakami C J, Burtner C R, Kennedy B K, et al. 2008. A method for high-throughput quantitative analysis of yeast chronological life span. J Gerontol A Biol Sci Med Sci 63A, 113-121.

Murphy M P. 2009. How mitochondria produce reactive oxygen species. Biochem J 417, 113.

Penninckx M J and Elskens M T. 1993. Metabolism and Functions of Glutathione in Micro-organisms. 34, 239-301.

Plauth A, Geikowski A, Cichon S, et al. 2016. Hormetic Shifting of Redox Environment by Pro-Oxidative Resveratrol Protects Cells Against Stress. Free Radic Biol Med.

Prasad S, Sung B and Aggarwal B B. 2012. Age-associated chronic diseases require ageold medicine: role of chronic inflammation. Prev Med 54 Suppl, S29-37.

Rahman I, Kode A and Biswas S K. 2006. Assay for quantitative determination of glutathione and glutathione disulfide levels using enzymatic recycling method. Nat Protoc 1, 3159-65.

Ramos-Gomez M, Olivares-Marin I K, Canizal-Garcia M, et al. 2017. Resveratrol induces mitochondrial dysfunction and decreases chronological life span of Saccharomyces cerevisiae in a glucose-dependent manner. J Bioenerg Biomembr.

Rebrin I, Kamzalov S and Sohal R S. 2003. Effects of age and caloric restriction on glutathione redox state in mice. Free Radical Biology and Medicine 35, 626-635.

Rockenfeller P and Madeo F. 2010. Ageing and eating. Biochim Biophys Acta 1803, 499506.

Schüller C, Brewster J L, Alexander M R, et al. 1994. The HOG pathway controls osmotic regulation of transcription via the stress response element (STRE) of the Saccharomyces cerevisiae CTT1 gene. EMBO J 13, 4382-4389.

Sharma S, Singh R, Kaur M, et al. 2010. Late-onset dietary restriction compensates for agerelated increase in oxidative stress and alterations of HSP 70 and synapsin 1 protein levels in male Wistar rats. Biogerontology 11, 197-209.

Tang L, Wang W, Zhou W, et al. 2015. Three-pathway combination for glutathione biosynthesis in Saccharomyces cerevisiae. Microb Cell Fact 14, 139.

Taylor E R, Hurrell F, Shannon R J, et al. 2003. Reversible glutathionylation of complex I increases mitochondrial superoxide formation. J Biol Chem 278, 19603-10.

Tietze F. 1969. Enzymic method for quantitative determination of nanogram amounts of total and oxidized glutathione: applications to mammalian blood and other tissues. Anal Biochem. 27, 502-22.

Vander Jagt D L, Robinson B, Taylor K K, et al. 1992. Reduction of trioses by NADPHdependent aldo-keto reductases. Aldose reductase, methylglyoxal, and diabetic complications. J Biol Chem. 267, 4364-9. 
Walsh M E, Shi Y and Van Remmen H. 2014. The effects of dietary restriction on oxidative stress in rodents. Free Radic Biol Med 66, 88-99.

Wei M, Brandhorst S, Shelehchi M, et al. 2017. Fasting-mimicking diet and markers/risk factors for aging, diabetes, cancer, and cardiovascular disease. Sci Transl Med 15, eaai8700.

Weinberger M, Mesquita A, Carroll T, Marks, L., , et al. 2010. Growth signaling promotes chronological aging in budding yeast by inducing superoxide anions that inhibit quiescence. Aging (Albany NY) 2, 709-726.

\section{Figure legends}

Figure 1. Effect of glucose concentration on the chronological life span and cellular bioenergetics of $S$. cerevisiae. a) The chronological life span of $S$. cerevisiae was measured in SC minimal media supplemented with 0.5 or $10 \%$ of glucose; b) the $\mathrm{H}_{2} \mathrm{O}_{2}$ release was measured by Amplex Red kit in $S$. cerevisiae cells grown in YPD medium supplemented with 0.5 or $10 \%$ of glucose; c-e) the oxygen consumption was measured at basal state and maximal respiratory capacity in $S$. cerevisiae cells grown in YPD medium supplemented with 0.5 or $10 \%$ of glucose. The results represent mean values \pm SEM from 3-5 independent experiments, which includes mean values of 3 technical repetitions. Statistical analyses were performed using two-tailed unpaired Student $t$-test.

Figure 2. Effect of glucose concentration and mutation of the gene GSH1 in the total glutathione of $S$. cerevisiae. Total glutathione was quantified by recycling assay in the WT and gsh $1 \Delta$ strains grown in YPD media supplemented with $0.5,2$ or $10 \%$ of glucose. Statistical analyses were performed using two-tailed unpaired Student $t$-test.

Figure 3. Influence of glucose concentration and mutation of the gene GSH1 on the $\mathrm{H}_{2} \mathrm{O}_{2}$ release of $S$. cerevisiae. The Amplex Red kit was used in the WT and gsh $1 \Delta$ strains grown in YPD medium supplemented with $0.5,2$ or $10 \%$ of glucose to measure the $\mathrm{H}_{2} \mathrm{O}_{2}$ release. Results represent mean values \pm SEM from 5 independent experiments. Statistical analyses were performed using two-tailed unpaired Student $t$-test.

Figure 4. Effect of glucose concentration and mutation of the gene GSHI in the chronological life span of $S$. cerevisiae. The chronological life span of WT and gshlA strains was measured in YPD medium supplemented with $0.5,2$ or $10 \%$ of glucose. The area under curve (AUC) survival was computed from the data of survival percentage vs. time using the trapezoid rule in the GraphPad Prism 6.00 for Macintosh. The results represent mean values \pm SEM from 4 independent experiments, which includes mean values of 3 technical repetitions. Statistical analyses were performed using two-tailed unpaired Student $t$-test.

Figure 5. Influence of sorbitol, glutathione supplementation, and mutation of the gene GSH1 in the chronological life span of S. cerevisiae. The chronological life span of WT and gsh $1 \Delta$ strains was measured in YPD medium supplemented with $0.5 \%$ glucose (glu) plus $9.5 \%$ sorbitol (sor) and $10 \%$ of glucose supplemented with $100 \mu \mathrm{M}$ glutathione (GSH). The area under curve (AUC) survival was computed from the data of survival percentage vs. 
time using the trapezoid rule in the GraphPad Prism 6.00 for Macintosh. The results represent mean values \pm SEM from 6 independent experiments. Statistical analyses were performed using two-tailed unpaired Student $t$-test.

Figure 6. Influence of glucose concentration, sorbitol, and mutation of the gene GSHI in the mitochondrial respiration of $S$. cerevisiae. The oxygen consumption was measured at basal state and maximal respiratory capacity in $S$. cerevisiae in exponential phase and chronologically aged grown in YPD medium supplemented with $0.5,2$ or $10 \%$ of glucose (glu) and $0.5 \%$ glucose plus $9.5 \%$ sorbitol (sor). The results represent mean values \pm SEM from 4 independent experiments, which includes mean values of 3 technical repetitions. Statistical analyses were performed using two-tailed unpaired Student $t$-test.

Figure 7. Effect of glucose concentration and mutation of the gene GSHI in the GSH/GSSG ratio of $S$. cerevisiae. GSH/GSSG ratio was quantified by recycling assay in the WT and gshl $\Delta$ strains grown in YPD media supplemented with 0.5 or $10 \%$ of glucose. Statistical analyses were performed using two-tailed unpaired Student $t$-test. 

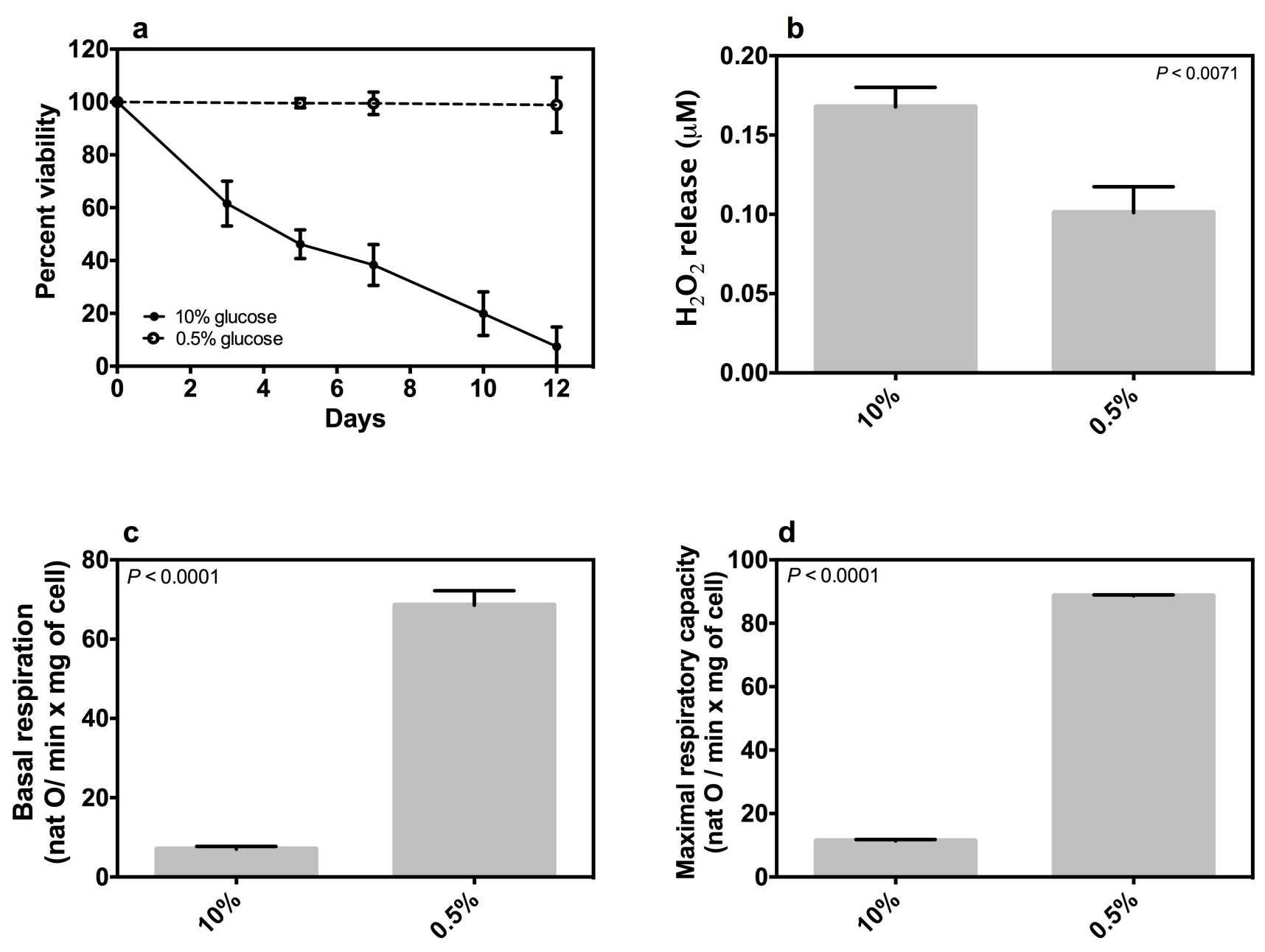


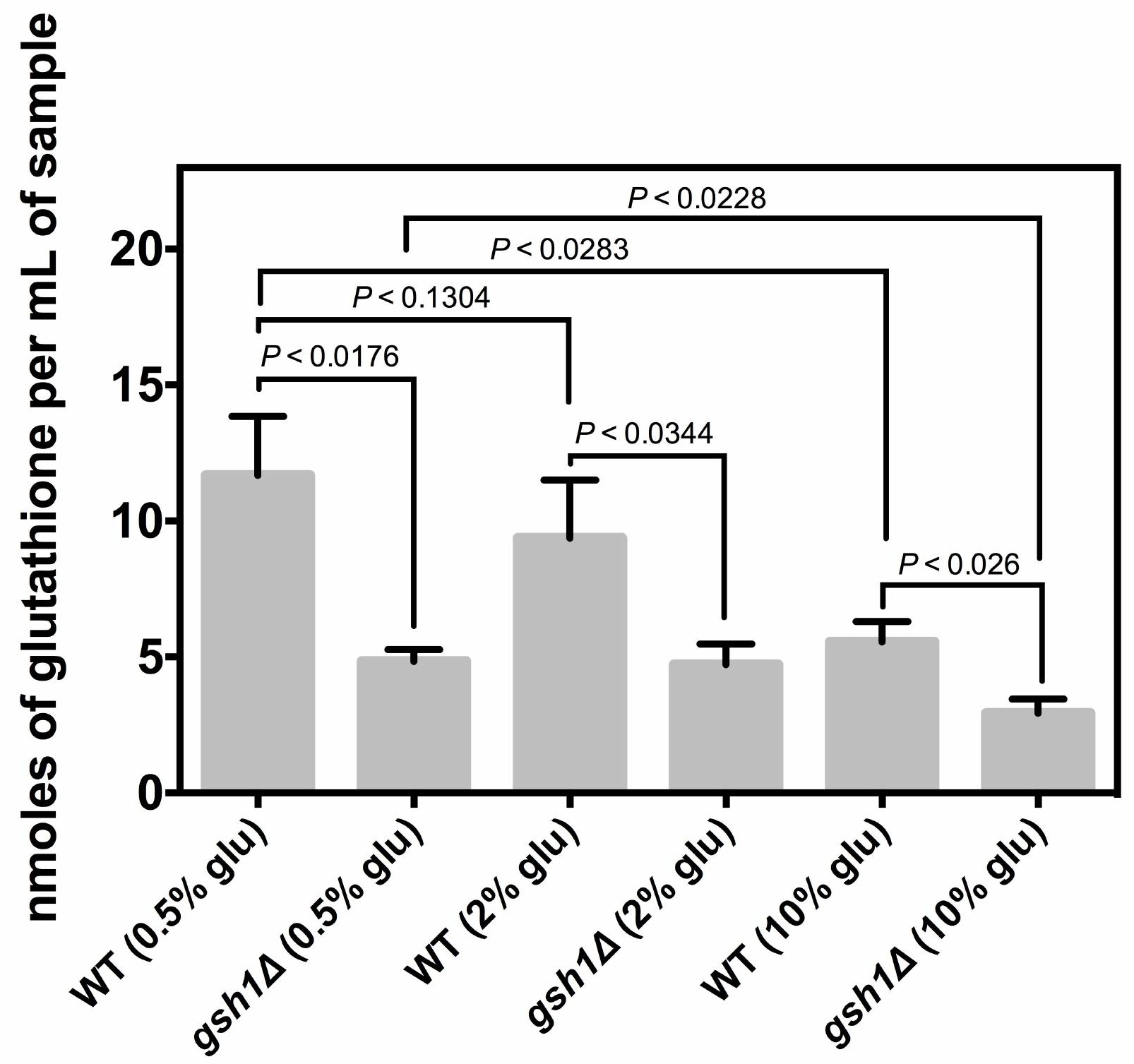



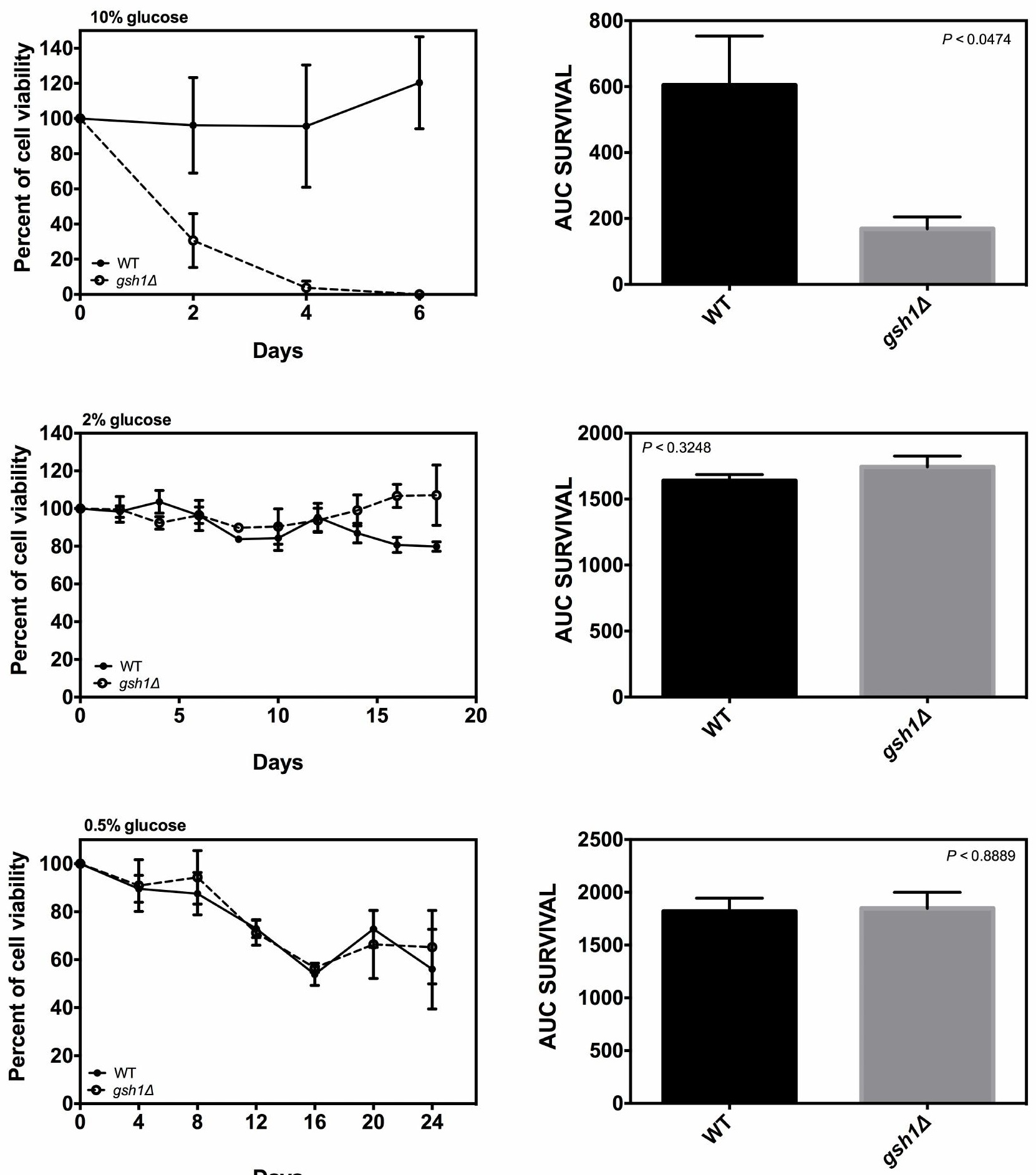

Days 

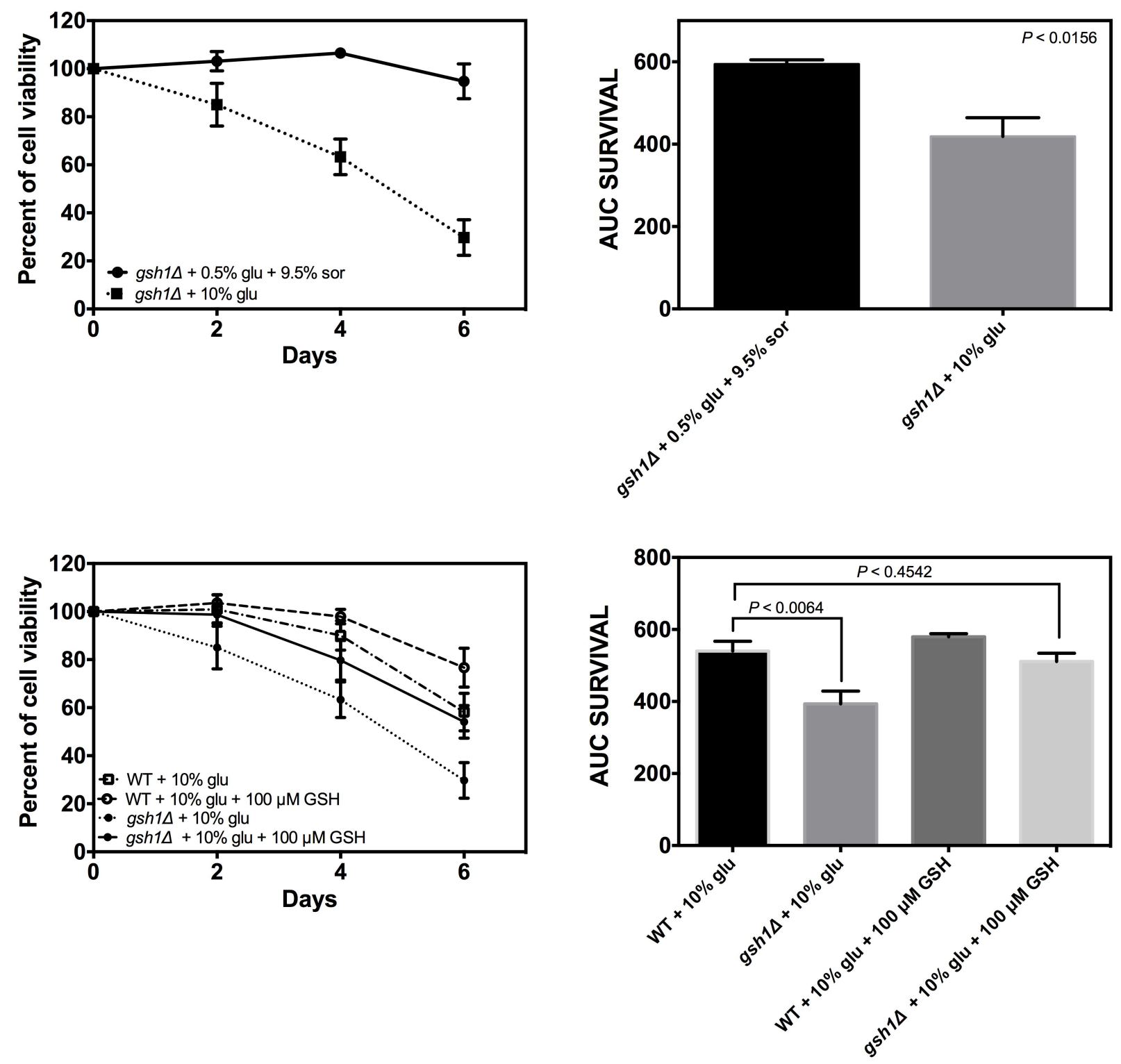


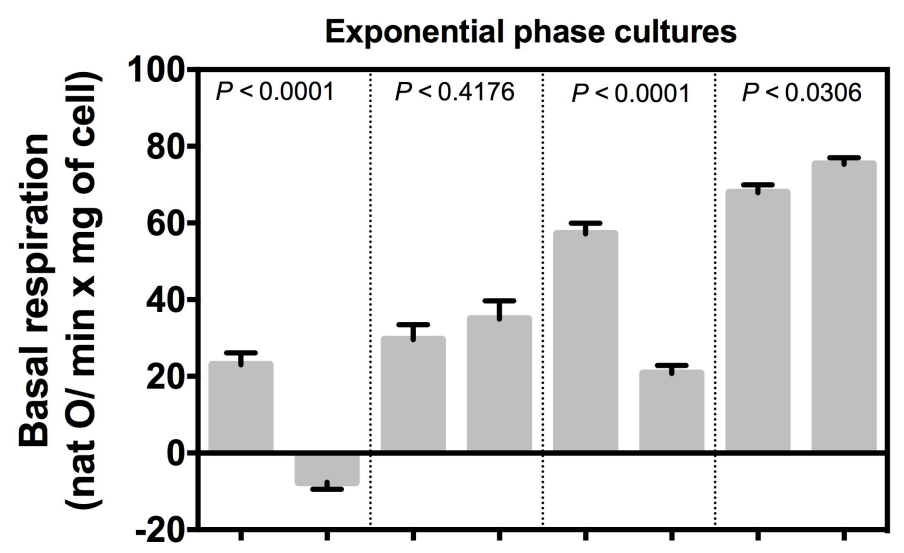

Chronologically aged cultures
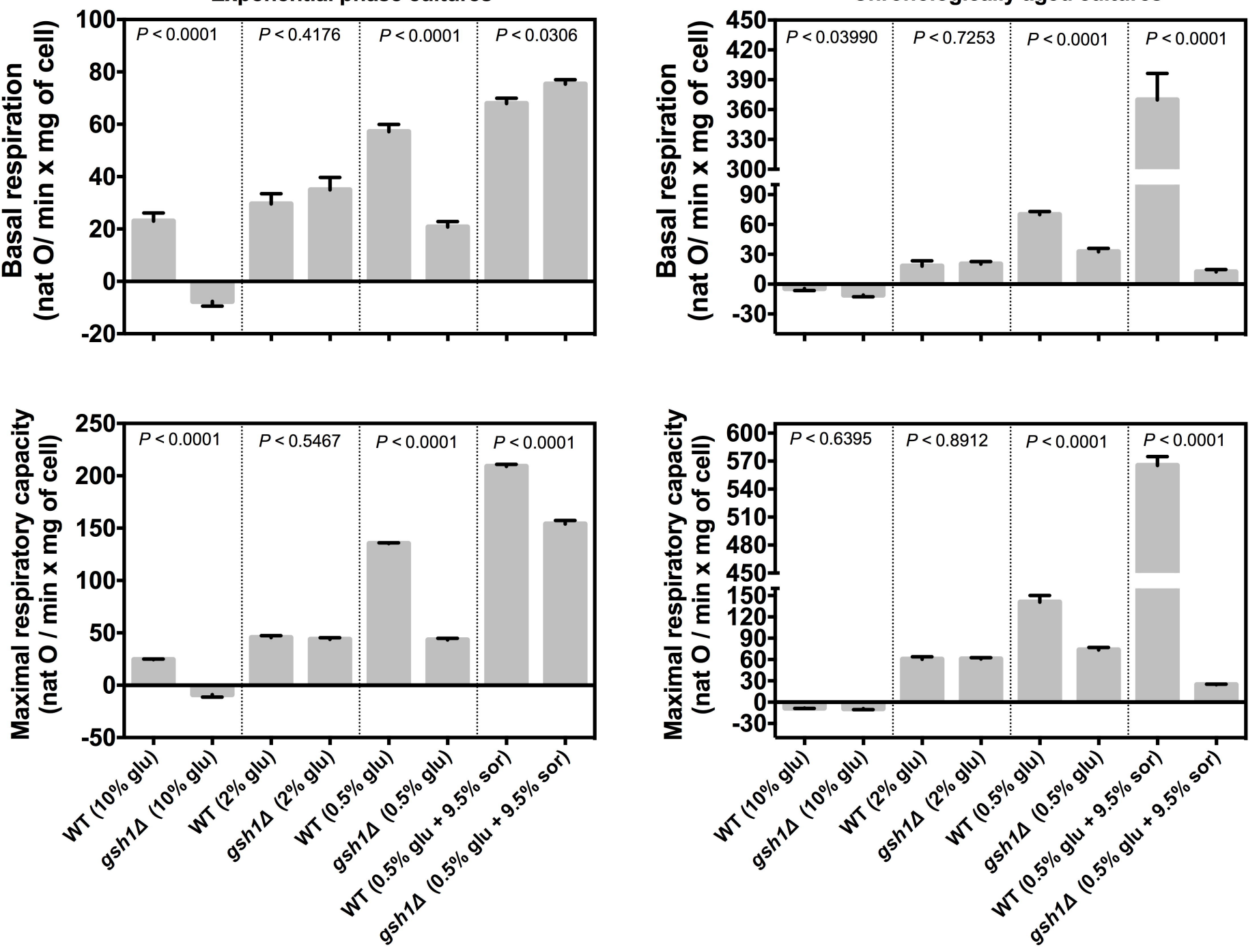
